\title{
Spectral matrix analysis for detection of polarized wave arrivals and its application to seismic reflection studies using local earthquake data
}

\author{
Hirokazu Moriya \\ School of Engineering, Tohoku University, 6-6-04 Aramaki, Aza Aoba, Aoba-ku, Sendai 980-8579, Japan \\ (Received November 27, 2008; Revised July 9, 2009; Accepted September 1, 2009; Online published January 18, 2010)
}

\begin{abstract}
Local earthquakes observed at Sendai, Japan, were analyzed to confirm the validity of a method of polarization analysis using the spectral matrix of seismic wave and its application to seismic reflection studies of the crust using local earthquake data. Reflectors (Bright spots) are known below the Nagamachi-Rifu fault, which caused an M 5.0 class event in 1998. Polarization analysis was applied to earthquake data in and around the fault. Use of the Z-parameter, which is defined using the eigenvalues of the spectral matrix and a statistical value representing the confidence level for the detection of the arrival of polarized waves, allowed detection of linearly and elliptically polarized waves in coda waves. The $Z$-parameter was also used to image the reflectors by using a migration technique that assumes the $P \times P$ and $S \times S$ reflection waves travel through a multi-layered velocity structure. Distinct reflectors were detected at depths of around $10 \mathrm{~km}, 14 \mathrm{~km}, 17 \mathrm{~km}, 21-26 \mathrm{~km}, 35 \mathrm{~km}$ and $40 \mathrm{~km}$, that is, from deeper than the fault and the Moho. This study demonstrated the feasibility of using the spectral matrix of three-component seismic signal to detect polarized waves and to image reflectors in the earth's crust and upper mantle.
\end{abstract}

Key words: Spectral matrix, seismic reflection, multiplet, migration.

\section{Introduction}

In 1998, an earthquake of magnitude 5.0 occurred beneath Sendai in the Miyagi district of Japan. There has been much research into the mechanism of this earthquake because it occurred in the deepest part of an active fault (the Nagamachi-Rifu fault) underlying the city. The geometry of the fault and the geological structure beneath the fault have been investigated through the analysis of earthquake data from in and around the fault, and by observations of the responses to explosive and vibrator sources at a smallaperture seismic array (e.g., Imanishi et al., 2002). Hori et al. (1999) revealed the existence of reflected $P \times P$ and $S \times S$ waves in the waveforms of aftershocks of the magnitude 5.0 event. Hasegawa et al. (2000) reported that such reflected phases have been extensively observed in the middle crust of the northeastern Japan arc. Umino et al. (2002) investigated the reflected waves in detail and estimated the depth and dip angle of the mid-crustal thin reflector. They estimated its depth to be from 15 to $21 \mathrm{~km}$ beneath the magnitude 5.0 event, and suggested that the existence of melt material and water at that depth would provide a plausible explanation for the reflectors (bright spots). Bright spots can usually be identified from reflected and converted waves in coda wave, and the detection of such the waves is important to understand their location and physical properties and also to elucidate their relationship to the earthquake occurrence.

The clear identification of reflected and converted waves is often difficult, even when the seismic signals with high

Copyright (c) The Society of Geomagnetism and Earth, Planetary and Space Sciences (SGEPSS); The Seismological Society of Japan; The Volcanological Society of Japan; The Geodetic Society of Japan; The Japanese Society for Planetary Sciences; TERRAPUB signal-to-noise ratios are detected, because the amplitude and amplitude ratios of detected three-component signal provides insufficient data to determine the arrival times and modes of the recorded waves. Analysis of the particle motions of seismic waves is an effective method to identify these waves, and several methods of polarization analysis have been proposed (e.g., Flinn, 1965; Benhama et al., 1988; Moriya et al., 1994; Moriya and Niitsuma, 1996; Greenhalgh et al., 2005; Diallo et al., 2006; Kulesh et al., 2007). Information about polarization can be used for various subsurface measurements; it has been applied to imaging of subsurface structures in geothermal fields (e.g., Soma et al., 1997, 2002, 2007).

Spectral matrix analysis is a polarization analysis method based on principal-component analysis in the time and frequency domains. This method can detect the arrival time of linearly or elliptically polarized waves by using the criterion of confidence levels. Through the analysis of crustal earthquakes, this method has successfully identified waves that may be $P \times P$ and $S \times S$ reflections from the upper and lower mantle, which suggests that polarization analysis using the spectral matrix is applicable to seismic reflection studies based on earthquake data (Moriya, 2008).

In this paper, I introduce a polarization analysis method that uses the spectral matrix (SPM) and analyze local earthquakes that occurred beneath Sendai City in northeastern Japan to confirm the feasibility of using SPM analysis and to investigate the reflectors observed below the active fault. This study demonstrates the feasibility of using SPM analysis in the analysis of earthquakes. 


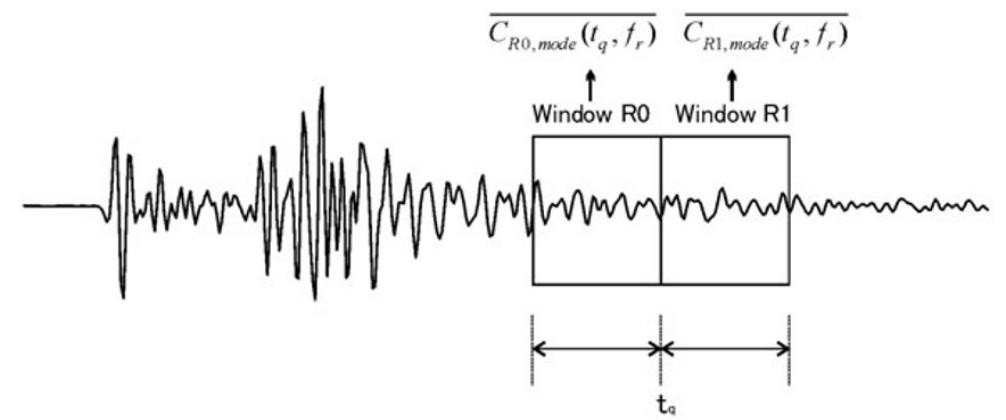

Evaluation of the difference of the correlation before and after $t_{q}$

$$
Z_{\text {mode }}\left(t_{q}, f_{r}\right)=\sqrt{(2 m+1) n-3}\left(Z_{T, \text { mode }}\left(\overline{C_{R 1, \text { mode }}\left(t_{q}, t_{r}\right)}\right)-Z_{T, \text { mode }}\left(\overline{C_{R 0, \text { mode }}\left(t_{q}, t_{r}\right)}\right)\right)
$$

Fig. 1. Concept of the $Z$-parameter. The $Z$-parameter evaluates the difference of $3 \mathrm{D}$ cross-correlations, which are calculated using the eigenvalues of the spectral matrix of the three-component signal at two independent time windows (window $R 0$ and $R 1$ ), before and after time $t_{q}$.

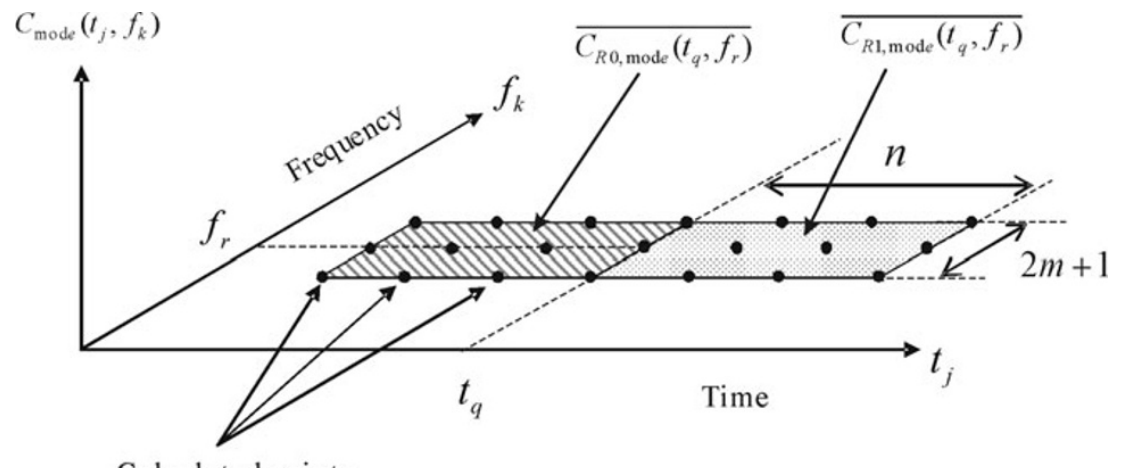

Calculated points

Fig. 2. Concept of mean values in the time-frequency domain (Moriya, 2008). $\overline{C_{R 0 \text {,mode }}\left(t_{q}, f_{r}\right)}$ and $\overline{C_{R 1} \text {,mode }\left(t_{q}, f_{r}\right)}$ are the mean values of $C_{\text {mode }}\left(t_{q}, f_{r}\right)$ for $(2 m+1) n$ samples taken from $C_{\text {mode }}\left(t_{q}, f_{r}\right)$ over a representative time $t_{q}$ and frequency $f_{r}$, where $t_{q}$ is the boundary time between two moving time windows used for calculating the spectral matrices.

\section{Detection of Reflected Waves Using the Z- Parameter in Polarization Analysis}

The arrivals of linearly or elliptically polarized waves can be detected by SPM analysis (Moriya and Niitsuma, 1996; Moriya, 2008). Here I define a statistical parameter that represents the confidence level for the detection of the arriving waves.

The spectral matrix is defined as a function of time and frequency as follows:

$$
\mathbf{S}_{\mathrm{p}}\left(t_{j}, f_{k}\right)=\left(\begin{array}{ccc}
S_{x x}\left(t_{j}, f_{k}\right) & S_{x y}\left(t_{j}, f_{k}\right) & S_{x z}\left(t_{j}, f_{k}\right) \\
S_{y x}\left(t_{j}, f_{k}\right) & S_{y y}\left(t_{j}, f_{k}\right) & S_{y z}\left(t_{j}, f_{k}\right) \\
S_{z x}\left(t_{j}, f_{k}\right) & S_{z y}\left(t_{j}, f_{k}\right) & S_{z z}\left(t_{j}, f_{k}\right)
\end{array}\right),
$$

where $S_{a a}\left(t_{j}, f_{k}\right)(a=x, y, z)$ are the power spectra; $S_{a b}\left(t_{j}, f_{k}\right)(a, b=x, y, z, a \neq b)$ are the cross-spectra, which are calculated using time series from a finite time window of the three-component signal; $t_{j}$ is a representative time showing the position of the moving time window; and $f_{k}$ denotes the frequency (Moriya and Niitsuma, 1996). The spectral matrix is generally complex but is always a Hermitian matrix, and is considered to be a covariance matrix in both the time and frequency domains (Moriya et al., 1994). The linearity and ellipticity of the polarization can be evaluated by using the eigenvalues $\left(\lambda_{i}\right)$ of the spectral matrix. I introduce two parameters to characterize the par- ticle motion of the waves as follows (Samson, 1973; Benhama et al., 1988; Soma et al., 1997; De Franco and Musacchio, 2001):

$$
\begin{aligned}
& C_{\text {Linear }}\left(t_{j}, f_{k}\right)=\frac{\left(\lambda_{1}-\lambda_{2}\right)^{2}+\left(\lambda_{1}-\lambda_{3}\right)^{2}+\left(\lambda_{2}-\lambda_{3}\right)^{2}}{2\left(\lambda_{1}+\lambda_{2}+\lambda_{3}\right)^{2}}, \\
& C_{\text {Elliptical }}\left(t_{j}, f_{k}\right)=1-\frac{2 \lambda_{3}}{\lambda_{1}+\lambda_{2}},
\end{aligned}
$$

where $\lambda_{i}=\lambda_{i}\left(t_{j}, f_{k}\right)\left(\lambda_{1}>\lambda_{2}>\lambda_{3}, i=1,2,3\right)$ are eigenvalues, and $C_{\text {Linear }}\left(t_{j}, f_{k}\right)$ and $C_{\text {Elliptical }}\left(t_{j}, f_{k}\right)$ are parameters that characterize linearity and ellipticity, respectively. I introduce a method that uses two moving time windows and has the ability to distinguish the arrival times of different coherent waves that closely overlap in time but have different frequency contents.

I introduce a concept of the statistical test for difference between two correlation functions to judge the arrivals of polarized waves statistically, I define the following function as the $Z$-parameter:

$$
\begin{aligned}
& Z_{\text {mode }}\left(t_{q}, f_{r}\right)= \sqrt{(2 m+1) n-3}\left(z_{T, \text { mode }} \overline{\left(C_{R 1, \text { mode }}\left(t_{q}, f_{r}\right)\right)}\right. \\
&\left.-z_{T, \text { mode }} \overline{\left(C_{R 0 \text {, mode }}\left(t_{q}, f_{r}\right)\right)}\right) \\
&(\text { mode }=\text { Linear or Elliptical }),
\end{aligned}
$$


where

$$
\begin{aligned}
& z_{T, \text { mode }} \overline{\left(C_{R 0}\left(t_{q}, f_{r}\right)\right)}=\frac{1}{2} \log \left(\frac{1+\overline{C_{R 0, \text { mode }}\left(t_{q}, f_{r}\right)}}{1-\overline{C_{R 0, \text { mode }}\left(t_{q}, f_{r}\right)}}\right) \\
& z_{T, \text { mode }} \overline{\left(C_{R 1}\left(t_{q}, f_{r}\right)\right)}=\frac{1}{2} \log \left(\frac{1+\overline{C_{R 1, \text { mode }}\left(t_{q}, f_{r}\right)}}{1-\overline{C_{R 1, \text { mode }}\left(t_{q}, f_{r}\right)}}\right)
\end{aligned}
$$

The subscript "mode" indicates the polarization type, either "Linear" or "Elliptical", referring to linear or elliptical polarization, respectively. $\overline{C_{R 0 \text {,mode }}\left(t_{q}, f_{r}\right)}$ and $\overline{C_{R 1 \text {,mode }}\left(t_{q}, f_{r}\right)}$ are the mean values of $C_{\text {mode }}\left(t_{j}, f_{k}\right)$ for $(2 m+1) n$ samples taken from $C_{\text {mode }}\left(t_{j}, f_{k}\right)$ over a representative time $t_{q}$ and with a frequency $f_{r}$, where $t_{q}$ is the boundary time between the two moving time windows used for calculating the spectral matrices, $m$ is the number of sample frequencies, and $n$ is the number of discrete sample times used to calculate the mean values as shown in Figs. 1 and 2 (Moriya and Niitsuma, 1996; Moriya, 2008). $\overline{C_{R 0 \text {,mode }}\left(t_{q}, f_{r}\right)}$ is the value before time $t_{q}$ and $\overline{C_{R 1 \text {, mode }}\left(t_{q}, f_{r}\right)}$ is the value after time $t_{q}$ (Fig. 1), and they have a value ranging from 0 to 1 according to linearity and ellipticity. Then, we can regard $\overline{C_{R 0 \text {,mode }}\left(t_{q}, f_{r}\right)}$ and $\overline{C_{R 1 \text {, mode }}\left(t_{q}, f_{r}\right)}$ as a function which represents correlation even though the function ranges from 0 to 1 and only indicates positive correlation. The parameters $z_{T \text {,mode }} \overline{\left(C_{R 0}\left(t_{q}, f_{r}\right)\right)}$ and $z_{T \text {,mode }} \overline{\left(C_{R 1}\left(t_{q}, f_{r}\right)\right)}$ follow an approximately normal distribution as a result of the transformation of $\overline{C_{R 0 \text {,mode }}\left(t_{q}, f_{r}\right)}$ and $\overline{C_{R 1 \text {,mode }}\left(t_{q}, f_{r}\right)}$ by Fisher's transformation (Eqs. (5) and (6)), where the parameters $z_{T \text {,mode }} \overline{\left(C_{R 0}\left(t_{q}, f_{r}\right)\right)}$ and $z_{T \text {,mode }} \overline{\left(C_{R 1}\left(t_{q}, f_{r}\right)\right)}$ follows a one-sided normal distribution because $\overline{C_{R 0 \text {,mode }}\left(t_{q}, f_{r}\right)}$ and $\overline{C_{R 1 \text {, mode }}\left(t_{q}, f_{r}\right)}$ represents only the positive value. Therefore, we could assume that the parameter $Z_{\text {mode }}\left(t_{q}, f_{r}\right)$ also has an approximately normal distribution.

$\overline{C_{R 0 \text {,mode }}\left(t_{q}, f_{r}\right)}$ and $\overline{C_{R 1 \text {,mode }}\left(t_{q}, f_{r}\right)}$ show the degree of polarization before and after a representative time $t_{q}$, and a statistical test can be introduced to judge whether the difference between $\overline{C_{R 0 \text {,mode }}\left(t_{q}, f_{r}\right)}$ and $\overline{C_{R 1 \text {, mode }}\left(t_{q}, f_{r}\right)}$ is significant. When a polarized wave with frequency $f_{r}$ arrives at time $t_{q}$ in the noise signal, the difference between $\overline{C_{R 0 \text {,mode }}\left(t_{q}, f_{r}\right)}$ and $\overline{C_{R 1 \text {,mode }}\left(t_{q}, f_{r}\right)}$ becomes larger and $Z_{\text {mode }}\left(t_{q}, f_{r}\right)$ has a peak.

Significance levels are commonly used in statistical tests to determine whether to reject or accept the null hypothesis. The significance level $\alpha$ is defined as follows:

$$
\alpha=\int_{z(\alpha)}^{+\infty} P(z) d z,
$$

where $P(z)$ has a standard normal distribution in which the random variable $z$ has the values of $Z_{\text {mode }}\left(t_{q}, f_{r}\right)$. If the following inequality is satisfied,

$$
Z_{\text {mode }}\left(t_{\text {arrival }}, f_{\text {arrival }}\right)>z(\alpha),
$$

then the null hypothesis which assumes the linearity and ellipticity are not changed before and after time $t_{\text {arrival }}$ is rejected under the significance level $\alpha$, and the hypothesis that a wave with linear or elliptical polarization has arrived is accepted, where the arrival of the polarized wave is estimated to have the significance level $\alpha$. If $z(\alpha)$ is 1.96 , then the arrival of a wave is detected with a confidence level of $97.5 \%$ under a one-sided testing. In practice, the value of $Z_{\text {mode }}\left(t_{q}, f_{r}\right)$ indicates a wave arrival and the confidence level of its detection.

The detectability of the method has been evaluated using the synthetic three-component signal. Moreover, it has been confirmed that the method can detect the arrival times of linearly and elliptically polarized waves within 10 sampling points at signal-to-noise ratios of $-7 \mathrm{~dB}$, and that the center frequency and the length of the time window are important for detection of the arrival times of the polarized waves with high accuracy (Moriya, 2008).

\section{Application to Local Earthquakes}

I used the waveforms observed at station TU.KMF (Fig. 3), which is located above the Nagamachi-Rifu fault and is operated by Tohoku University, because they show the bright spots and other reflectors (e.g., Umino et al., 2002) and because I can compare my results with those of Umino et al. (2002). Table 1 shows the information on used earthquakes.

The 3D seismic wave subsurface velocity structure of northeastern Japan has been investigated by Nakajima et al. (2001), who identified low $V_{s}$ and large $V_{p} / V_{s}$ zones in the lower crust close to the uppermost mantle. Their estimated $V_{p} / V_{s}$ ratio implies that the high $V_{p} / V_{s}$ region below station TU.KMF is at a depth of around $23-48 \mathrm{~km}$. Umino et al. (2002) revealed that the planar $S$-wave reflector is shallower than this low $V_{s}$ and $V_{p} / V_{s}$ zone and showed that it is within a depth range of 15-21 km, just beneath the fault associated with the 1998 Sendai earthquake. Many other reflectors have also been located at these depths by the image station method of Horiuchi et al. (1988).

Figure 4 shows an example of waveforms from station TU.KMF and the calculated $Z$-parameters, where the threecomponent signal (U-D, E-W, N-S) and the parameters for linear and elliptical polarization are also shown. The symbols $\bigcirc$ and denote the arrivals of $P$ - and $S$-waves detected by the $Z$-parameters.

The Fourier transform was calculated by using an FFT algorithm, which requires less calculation time than other spectral estimation methods. A 0.3-s time window was used to ensure that the $Z$-parameters were large at the arrival times of the $P$ - and $S$-waves. In general, the time window should be long enough to estimate the spectrum with confidence, but in practice, the appropriate length should be determined with reference to the peak value of the $Z$ parameters for $P$ - and $S$-waves to ensure detection of reflected waves, because the seismic waves are contaminated by multiple reflections and random scatter. It is therefore difficult to theoretically determine an optimum time window.

Detectability was evaluated using a synthetic wave signal consisting of sinusoidal waves and random noise, where I prepared the synthetic three-component signal, which was produced by attenuated tone burst sinusoidal waves and a computer-generated random number, and the synthetic three-component signal comprised three linearly polarized 


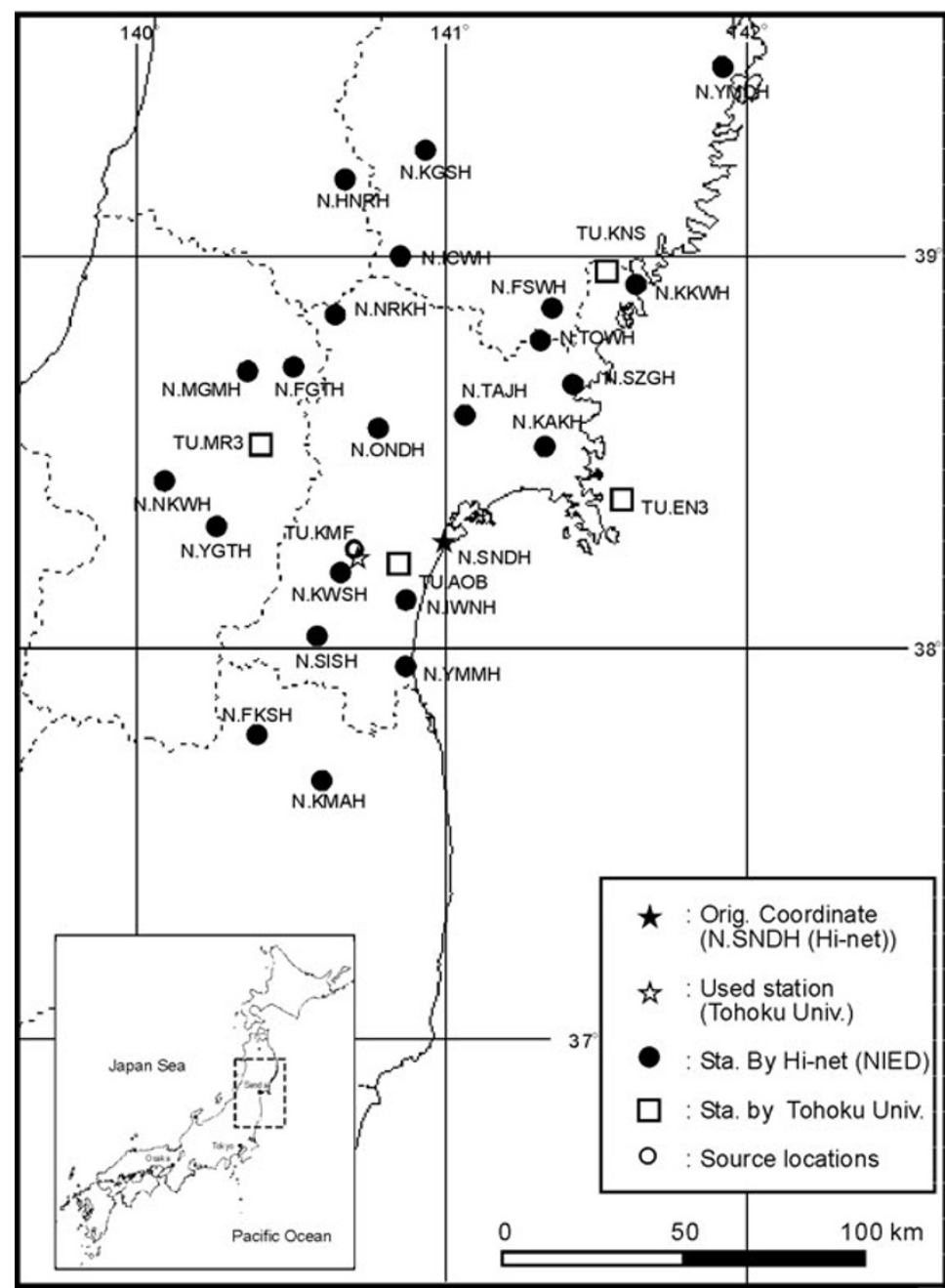

Fig. 3. Seismic observations stations in the area around Sendai District, northeastern Japan. The earthquakes observed at the station shown by the symbol $i$ were used in this study. The source locations of events used in this study are also plotted by open circles.

Table 1. Source locations of analyzed earthquakes.

\begin{tabular}{cccccc}
\hline Event No. & Date and time & $\begin{array}{c}\text { Longitude } \\
\text { (degrees) }\end{array}$ & $\begin{array}{c}\text { Latitude } \\
\text { (degrees) }\end{array}$ & $\begin{array}{c}\text { Depth } \\
(\mathrm{km})\end{array}$ & $M$ \\
\hline 1 & $2003 / 09 / 0710: 05: 06$ & 140.6920 & 38.2297 & 8.2 & 1.4 \\
2 & $2003 / 09 / 0711: 08: 31$ & 140.6982 & 38.2395 & 7.1 & 0.0 \\
3 & $2003 / 09 / 0714: 25: 12$ & 140.6897 & 38.2305 & 8.0 & 1.4 \\
4 & $2003 / 10 / 0311: 50: 05$ & 140.6938 & 38.2333 & 8.4 & 1.5 \\
\hline
\end{tabular}

waves and one elliptically polarized wave, each a sinusoidal wave with a frequency of $5 \mathrm{~Hz}$, assuming a sampling frequency of $100 \mathrm{~Hz}$, where the different signal-to-noise ratios $(S / N)$ were given to the three linearly polarized waves and the one elliptically polarized wave (Moriya, 2008). Then, I have evaluated the dependence of detectability on time window length and the center frequency for analysis, where I searched for an optimal time window length and the center frequency for analysis as the $Z$-parameter has a larger values responding to arrival of polarized waves. As the result, I chose a frequency band of $5-15 \mathrm{~Hz}$ and a time-window of $0.3 \mathrm{~s}$ for my calculation of the $Z$-parameter, also considering that the power spectral density of body waves is dominant from 10 to $15 \mathrm{~Hz}$. I included frequencies lower than $10 \mathrm{~Hz}$ because the use of lower frequency is favorable for the detection of reflection waves due to the less attenuation during the wave propagation. The length of time series for FFT was 128 points and the frequency resolution was $0.781 \mathrm{~Hz} /$ point, because the sampling frequency of the waveform is $100 \mathrm{~Hz}$.

The abovementioned conditions correspond to a center frequency of $10 \mathrm{~Hz}$ and $m$ and $n$ values of 6 and 5 , where $m$ is automatically determined by the frequency band width and the frequency resolution. That is the frequency band width is $10 \mathrm{~Hz}$ and the frequency resolution is $0.781 \mathrm{~Hz} /$ point, then $2 m+1=13$. The $n$ value of 5 was arbitrary given to calculate the average value along time axis.

In Fig. 4, the detected arrival time difference between 


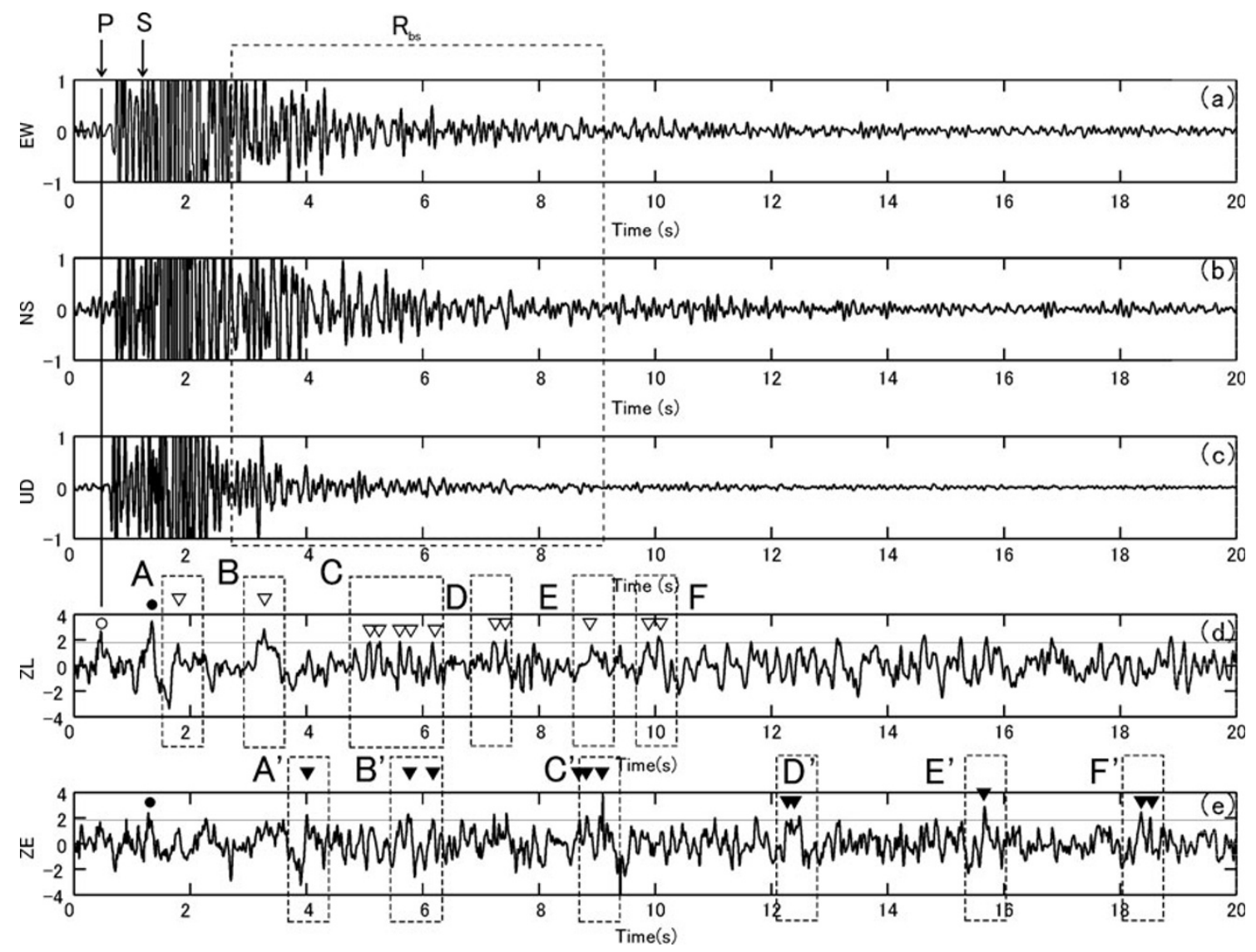

Fig. 4. Three-component waveforms (a) to (c) of an earthquake and calculated $Z$-parameters (d) and (e). The symbols $\bigcirc$ and $\bigcirc$ denote the arrivals of $P$ - and $S$-waves. The gray lines in (d) and (e) represent a $Z$-parameter value of 1.96 . The thick line represents the arrival time detected by the $Z$-parameter.

Table 2. Velocity stricture used for analysis (Nakajima et al., 2001).

\begin{tabular}{lcc}
\hline & $\begin{array}{c}V_{p} \\
(\mathrm{~km} / \mathrm{s})\end{array}$ & $\begin{array}{c}V_{s} \\
(\mathrm{~km} / \mathrm{s})\end{array}$ \\
\hline Upper crust (0-10 km depth) & 6.31 & 3.67 \\
Lower crust (11-25 km depth) & 6.85 & 3.93 \\
Uppermost mantle (26-40 km) & 7.90 & 4.57 \\
\hline
\end{tabular}

$P$ - and $S$-waves was about 0.835 s because the depth of the source was about $7 \mathrm{~km}-9 \mathrm{~km}$ and the observation point was almost directly above the seismic source. If the velocity structure is assumed to be a multi-layered structure as shown in Table 2 (Nakajima et al., 2001) and that the parameters $Z_{\text {Linear }}\left(t_{q}, f_{r}\right)$ and $Z_{\text {Elliptical }}\left(t_{q}, f_{r}\right)$ represent reflected $P$ - and $S$-waves, respectively, the $P \times P$ and $S \times S$ reflections from a depth of $15-21 \mathrm{~km}$ appear at a time within $R_{\mathrm{bs}}$, indicated by the large dashed rectangle of Fig. 4, and several wavelets with larger amplitudes are also evident. The gray lines on panels (d) and (e) of Fig. 4 ( $Z$-parameter $=1.96)$ indicate that the confidence of detection is more than $97.5 \%$ if the $Z$-parameter is larger than 1.96 .

The $P$ - and $S$-wave arrivals are clearly shown in Figs. 4(d) and (e). The $P$-wave arrival in Fig. 4(d) seems to be earlier, but the $P$-wave arrives at the time indicated by the open circle and a wave appears at EW component if we observe the details of $P$-wave onset in EW component. The reason the $Z$-parameter $\left(Z_{\text {Linear }}\left(t_{q}, f_{r}\right)\right)$ also shows a peak

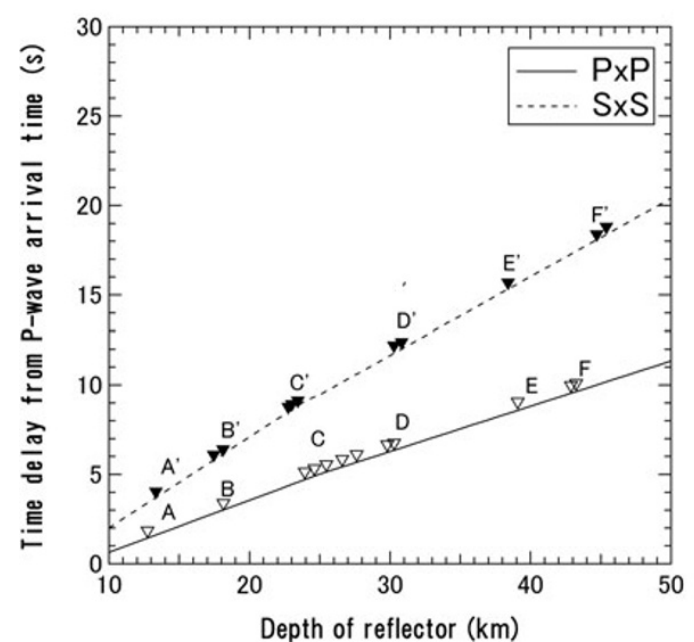

Fig. 5. Relationship between the time delay of the calculated reflected wave arrival time with respect to the $P$-wave arrival time and depth of reflector. The symbols $\nabla$ and $\boldsymbol{\nabla}$ as well as the letters A-D and $\mathrm{A}^{\prime}-\mathrm{D}^{\prime}$ denote the peaks shown in Fig. 4. The peaks of $Z$-parameters are considered to represent the reflected waves from the corresponding depths.

at the $S$-wave arrival time is that the $S$-wave has larger signal to noise ratio and the particle motion of the $S$-wave is rectilinear, rather than elliptical in this case, and this results in the parameter $Z_{\text {Linear }}\left(t_{q}, f_{r}\right)$ responding to the $S$-wave 
arrival. Several clear peaks can be seen in the regions A$\mathrm{E}$ and $\mathrm{A}^{\prime}-\mathrm{E}^{\prime}$ (indicated in Fig. 4 by symbols $\nabla$ and $\boldsymbol{\nabla}$, respectively); these peaks indicate the arrivals of polarized waves.

To investigate the expected arrivals of reflected waves, I calculated their theoretical travel times from arbitrary depths and compared them with the peaks of $Z$-parameters. Figure 5 shows the relationship between the time delay of the calculated reflected wave arrival with respect to the $P$ wave arrival time and the depth of the reflector, where the travel time was calculated assuming both $P \times P$ and $S \times S$ reflection waves within a multi-layered velocity structure. I first detected the peaks which is larger than the level show by a thick line and secondary detected the peaks in $Z_{\text {Elliptical }}\left(t_{q}, f_{r}\right)$ which would be corresponding to the peaks identified in $Z_{\text {Linear }}\left(t_{q}, f_{r}\right)$. Here, the symbols $\nabla$ and $\boldsymbol{\nabla}$ denote the $Z$-parameter peaks of Fig. 4 . It should be noted in Fig. 5 that the $Z$-parameter peaks seems to appear synchronously for both $Z_{\text {Linear }}\left(t_{q}, f_{r}\right)$ and $Z_{\text {Elliptical }}\left(t_{q}, f_{r}\right)$ with time delay due to the difference of $V_{p}$ and $V_{s}$, and the correspondence between $Z_{\text {Linear }}\left(t_{q}, f_{r}\right)$ and $Z_{\text {Elliptical }}\left(t_{q}, f_{r}\right)$ is indicated by capital letters $\mathrm{A}-\mathrm{E}$ and $\mathrm{A}^{\prime}-\mathrm{E}^{\prime}$. This result would imply that the reflected waves with both $P$ - and $S$-wave came from the same reflector and that the pair of peaks that was detected in both $Z_{\text {Linear }}\left(t_{q}, f_{r}\right)$ and $Z_{\text {Elliptical }}\left(t_{q}, f_{r}\right)$ had a time delay due to the different $P$ - and $S$-wave velocities.

On the other hand, other peaks appear both in $Z_{\text {Linear }}\left(t_{q}, f_{r}\right)$ and $Z_{\text {Elliptical }}\left(t_{q}, f_{r}\right)$, especially at the latter part of coda wave. One of the possible reasons is that there are real multi-layered reflectors, and another possibility is that the virtual peaks appear due to low signal to noise ratio. The value of $Z$-parameter represents the confidence for the arrivals of linearly or elliptically polarized waves, but the accuracy becomes lower at the portion of low signal to noise ratio such as latter part of coda wave. It is because the $Z$-parameter is a function following a stochastic process and the tendency to have an unexpected large value vary from true value at low signal to noise ratio increases due to the perturbation by noise.

\section{Imaging of Subsurface Structures}

The linearly and elliptically polarized wave components were detected using the $Z$-parameters, and were assumed to represent reflected waves. Umino et al. (2002) detected $P \times P$ and $S \times S$ reflection waves and reflectors below the Nagamachi-Rifu fault. Then, I next applied the $Z$ parameter to seismic reflection imaging. I used a simple migration to obtain the reflection images, one in which $P \times P$ and $S \times S$ reflected waves are considered to travel within a multi-layered velocity structure shown in Table 2 . The reason I used this simple migration approach is that I wanted to confirm whether the detected polarized components represent the reflected waves of the reflectors, and whether the $Z$-parameters are applicable for imaging subsurface structure as well as for the detection of reflected waves. In this context, a complex migration algorithm is not required.

Figure 6 shows the waveforms of events that were observed at station TU.KMF between 7 September and 3 October 2003. The waveforms of each are multiplet events for which source locations were adjacent. The use of multiplets

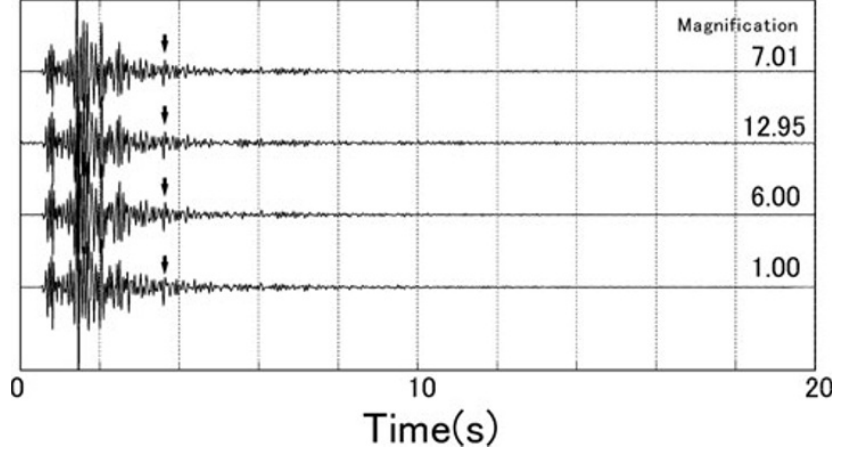

Fig. 6. Normalized E-W components of waveforms of four local earthquakes observed at station TU.KMF between 7 September and 3 October 2003. The amplitude of waveforms are normalized by using the fourth event, and the amplitude ratios of events relative to the fourth event are shown in the figure. The four events shown are multiplet events of similar waveform. The magnitudes of the events ranged from 1.5 to 0.0 .
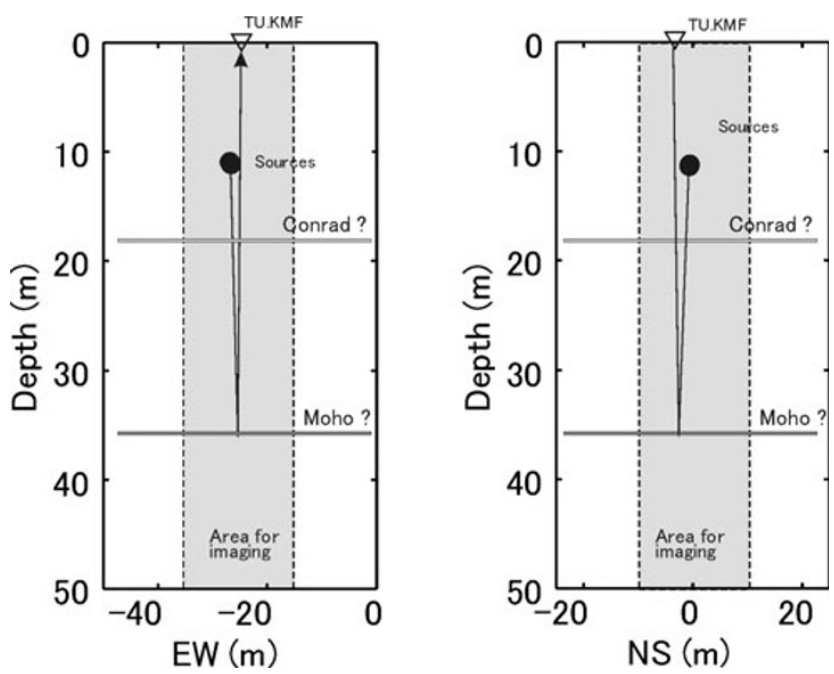

Fig. 7. Schematic view showing the relationship between observation stations and seismic source. The angles of incidence and reflection are close to zero for a horizontal reflector. The expected depths of the Conrad and Moho discontinuities are shown.

emphasizes the image of a reflector because the reflected waves appear at the same time with similar waveforms and the contrast between the reflection and background data is sharpened.

Figure 7 shows the schematic view that provides two perpendicular vertical cross sections which show the geometric relationships among source locations, observations, and the expected reflectors. The earthquakes selected as sources occurred almost directly below the recording station.

Figures 8(a) and (b) show the parameters $Z_{\text {Linear }}\left(t_{q}, f_{r}\right)$ and $Z_{\text {Elliptical }}\left(t_{q}, f_{r}\right)$ for four earthquakes recorded at station TU.KMF. Peaks appear at times corresponding to $P$ - and $S$-wave arrivals (symbols $\bigcirc$ and $\bigcirc$ ), and the alignment of these peaks suggests that other irregular peaks are caused by noise. One clear alignment of peaks can be identified in the coda wave (symbols $\nabla$ and $\boldsymbol{\nabla}$ ); these appear around 3.0 to $3.5 \mathrm{~s}$ after the $P$-wave arrival. This distinct phase can also be seen in the waveforms of Figs. 4 and 6 (regions B and $\mathrm{B}^{\prime}$ in Fig. 4 and as indicated by arrows in 

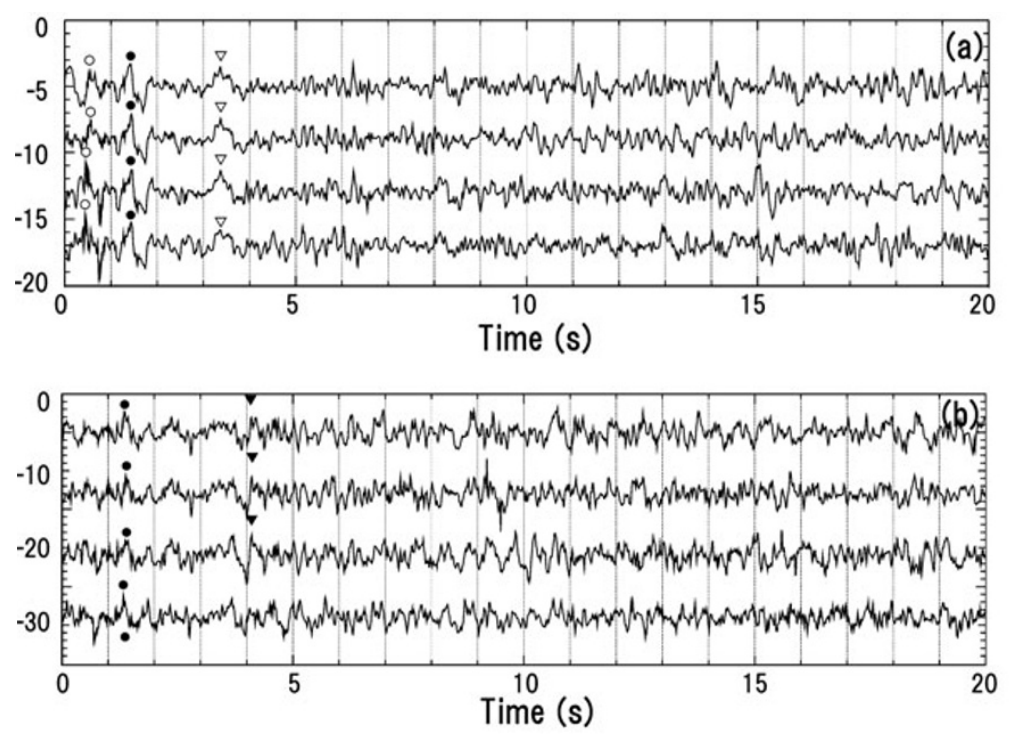

Fig. 8. Calculated $Z$-parameters for four earthquakes recorded at station TU.KMF between 7 September and 3 October 2003. (a) $Z_{\text {Linear }}\left(t_{q}, f_{r}\right)$ and (b) $Z_{\text {Elliptical }}\left(t_{q}, f_{r}\right)$.
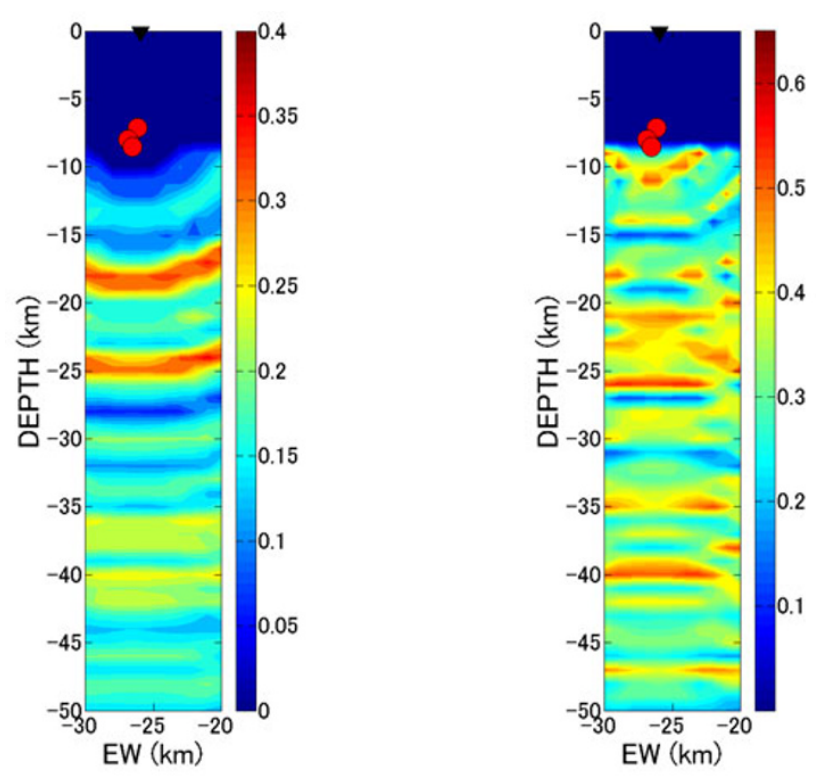

Fig. 9. Reflection images derived from (a) $Z_{\text {Linear }}\left(t_{q}, f_{r}\right)$ and (b) $Z_{\text {Elliptical }}\left(t_{q}, f_{r}\right)$ assuming $Z_{\text {Linear }}$ represents $P \times P$ and $Z_{\text {Elliptical }}$ represents $S \times S$. The closed circle denotes the source locations of analyzed events. The closed circles denote the source locations and the triangles denote the seismic observation point.

Fig. 6). Other researchers have confirmed the existence of these data in earthquake records (e.g. Umino et al., 2002). Their existence implies that the arrivals of polarized waves from reflectors and scatter points can be detected by $Z$ parameters.

The imaging was carried out as follows. First, virtual reflection points were assumed horizontally every $1 \mathrm{~km}$ within a range of $10 \mathrm{~km}$ in both the $\mathrm{E}-\mathrm{W}$ and N-S directions and vertically over a depth range of 0 to $50 \mathrm{~km}$. The theoretical arrival time of each reflected $P$ - and $S$-wave from each virtual reflection point was calculated, and the value of $Z_{\text {mode }}\left(t_{q}, f_{r}\right)$ at the time corresponding to the theoretical re- flection arrival time was assigned to the corresponding virtual reflection point. The same operation was performed for all earthquakes, and the values were accumulated at the virtual reflection points. Finally, the ensemble averages of the $Z_{\text {mode }}\left(t_{q}, f_{r}\right)$ related to each event were calculated at each virtual reflection point. This process is based on the same concept as the Kirchhoff migration method, which draws a reflection-time surface by using the amplitude of waves or reflection coefficients. In this study, amplitude is replaced by the parameter $Z_{\text {mode }}\left(t_{q}, f_{r}\right)$, which represents the confidence level of the polarized wave arrival.

Figures 9(a) and 9(b) show the reflection images derived from the parameters $Z_{\text {Linear }}\left(t_{q}, f_{r}\right)$ and $Z_{\text {Elliptical }}\left(t_{q}, f_{r}\right)$, assuming that the peaks in $Z_{\text {Linear }}\left(t_{q}, f_{r}\right)$ and $Z_{\text {Elliptical }}\left(t_{q}, f_{r}\right)$ represent the $P \times P$ and $S \times S$ reflection waves, respectively.

\section{Discussion}

According to the hypothesis discussed above, the value of a $Z$-parameter can be considered as the confidence level of detection. For instance in Fig. 9(a), the maximum value of the ensemble-averaged $Z$-parameters was 0.7 , and in this case the hypothesis that waves with linear or elliptical polarization have arrived is accepted with a confidence level of $50 \%$. Therefore, we can say that the value of $Z$-parameter also means the confidence level for detection of reflectors as well as for reflected waves, and that the confidence level is greater for larger values of the $Z$-parameter.

Both the $P$ - and $S$-wave are reflected under the condition that the reflection angle is larger than critical angle, even though they have different reflection coefficients, and both show reflectors at the same depths in processed seismic images. The several reflectors of high amplitude in Figs. 9(a) and 9 (b) are associated with the pairs of peaks shown in Fig. 5, indicated by $\mathrm{A}-\mathrm{E}$ and $\mathrm{A}^{\prime}-\mathrm{E}^{\prime}$. The difference of the reflector depths in panels (a) and (b) of Figs. 9 would be attributed to the velocity structure used. This difference is also apparent in Fig. 5 and the expected depths of reflectors are slightly different for $P \times P$ and $S \times S$, for instance, 
the open triangle at $\mathrm{A}$ is different from the closed triangle at $\mathrm{A}^{\prime}$ in the depth. On the other hand, the two high amplitude reflectors in Figs. 9(a) and 9(b) correspond to reflectors near the bottom of the Nagamachi-Rifu fault that have been identified by other researchers at depths of about 15-17 and $20-22 \mathrm{~km}$.

Understanding the structure below active faults is important. Sub-horizontal detachment is thought to be common in the deep extension of faults; slip on this part of the detachment surface might be responsible for earthquakes related to the active fault (e.g., Ikeda, 1992). This study suggests that the $Z$-parameter can be used to detect reflection waves through polarization analysis of three-component seismic signals and that it can also be used to image reflectors.

Because the $Z$-parameter is a function of frequency and a statistical value, the method presented here is robust when signal-to-noise ratios are poor. It could thus have an advantage over other methods in the detection of reflected waves within the coda wave. In addition, the ensemble average of $Z$-parameters calculated from several waveforms removes virtual images associated with noise, where peaks representing reflection waves may not be visible (Figs. 4, 8(a), and 8(b)). This may have advantages at deep levels where the structure is not readily apparent and where the signalto-noise ratio is low in the late part of the coda wave. In Figs. 9(a) and 9(b) reflectors are imaged at depths of around $10 \mathrm{~km}, 14 \mathrm{~km}, 17 \mathrm{~km}, 21-26 \mathrm{~km}, 35 \mathrm{~km}$ and $40 \mathrm{~km}$. The reflector at $35-36 \mathrm{~km}$ and $40 \mathrm{~km}$ likely represents the Moho.

The results of this study show that the method presented can detect reflected waves that are difficult to see with the naked eye, and that it is applicable to finding reflectors in the crust by using local earthquake data. It is important to note that the two $Z$-parameters $\left(Z_{\text {Linear }}\left(t_{q}, f_{r}\right)\right.$ and $\left.Z_{\text {Elliptical }}\left(t_{q}, f_{r}\right)\right)$ are defined to separately identify the arrivals of $P$ - and $S$-wave components that have primarily linear and elliptical polarization. The arrival of an $S$ wave component with rectilinear polarization might be mistaken for the arrival of a $P$-wave component, as shown in Figs. 4(d) and (e). This means that we need to consider the reflected waves with both $P$ - and $S$-wave components (e.g., at least $P \times P$ and $S \times S$ ) when we migrate the $Z$-parameters to image reflectors, and in discussion of the positions of those reflectors. This is the limitation of this method that we can not exactly judge the reflection modes of arriving waves and need to assume $P \times P, S \times S, S \times P, S \times S$ and so on, because the $Z$-parameter only identifies the polarization type and the arrival times of detected waves. Therefore, we need to discuss how to delineate reflectors clearly overcoming the above mentioned limitation in the future. For instance, the reflectors could be delineated by stacking the all the results, which are obtained assuming $P \times P, S \times S$, $S \times P$ and $P \times S$ reflection, where the virtual images could be canceled and the possible reflectors could be emphasized through the effect of stacking.

In this study, I used data from only one observation station, and a simplified migration algorithm, because the purpose of the study was to confirm whether the $Z$-parameter derived from the spectral matrix can detect reflected seismic waves and to test the application of the method for seismic reflection studies based on local earthquake data. The use of multiple observation stations and a more realistic migration velocity model would refine the method and better define the reflectors identified.

\section{Conclusions}

I have proposed a method for the detection of arrival times of polarized seismic waves by using the spectral matrix. I applied the method to data from local earthquakes in the Sendai district, which is an area where bright spots and other reflectors have been identified under the NagamachiRifu fault by other researchers. My aim was to examine whether the method can be used to image subsurface reflectors. Multiplet events of similar waveform from around $10 \mathrm{~km}$ depth almost directly below a seismic observation station were analyzed, and $Z$-parameters, which are functions defined by eigenvalues of the spectral matrix, were used to derive confidence levels for the detection of the arrivals of polarized waves. The $Z$-parameter was used to image subsurface reflectors, and the obtained image was compared with reflectors detected by other researchers. Several reflectors were identified at depths just below the fault and in the region of the Moho discontinuity. The study demonstrated that polarized waves can be objectively detected by using the $Z$-parameter and that the parameter can be used in reflection seismic studies. This method of detection of polarized waves will be a useful tool for highly accurate and objective detection of polarized wave arrivals and also for seismic reflection studies based on earthquake data.

Acknowledgments. I thank the National Research Institute for Earth Science and Disaster Prevention (NIED) of Japan and Tohoku University for providing the earthquake waveform data used in this study. The data were downloaded from the NIED website (http://www.hinet.bosai.go.jp/). This research was partially supported by the Ministry of Education, Science, Sports and Culture, Grant-in-Aid for Scientific Research (B), No. 20360402.

\section{References}

Benhama, A., C. Cliet, and M. Dubesset, Study and applications of spatial directional filtering in three-component recordings, Geophys. Prospect., 36, 591-613, 1988.

De Franco, R. and G. Musacchio, Polarization filter with singular value decomposition, Geophysics, 66, 932-938, 2001.

Diallo, M., M. Kulesh, M. Holschneider, K. Kurennaya, and F. Scherbaum, Instantaneous polarization attributes based on an adaptive approximate covariance method, Geophysics, 71, V99-V104, 2006.

Flinn, E. A., Signal analysis using rectilinearity and direction of particle motion, Proc. IEEE, 59, 1234-1237, 1965.

Greenhalgh, S., L. M. Mason, and B. Zhou, An analytical treatment of single station triaxial seismic direction finding, J. Geophys. Eng., 2, 815, doi:10.1088/1742-2132/2/1/002, 2005.

Hasegawa, A., A. Yamamoto, N. Umino, S. Miura, S. Horiuchi, D. Zhao, and H. Sato, Seismic activity and deformation process of the crust within the overriding plate in the northeastern Japan subduction zone, Tectonophysics, 319, 225-239, 2000.

Hori, S., N. Umino, Y. Asano, and A. Hasegawa, S-wave reflectors in the crust of northeastern Japan, Programme and Abstracts, The Seismological Society of Japan, 1999 Fall Meeting, p. 140, 1999.

Horiuchi, S., A. Hasegawa, A. Takagi, A. Ito, M. Suzuki, and H. Kameyama, Mapping of a melting zone near Mt. Nikko-Shirane in northern Kanto, Japan, as inferred from $\mathrm{S} \times \mathrm{P}$ and $\mathrm{S} \times \mathrm{S}$ reflections, Tohoku Geophys. J., 31, 43-55, 1988.

Ikeda, Y., Japanese thrust faults: A possibility of flake tectonics, Monthly Chikyu, Extra 5, 1992 (in Japanese).

Imanishi, K., H. Ito, Y. Kuwahara, Y. Mamada, T. Yokokura, N. Kano, K. Yamaguchi, and A. Tanaka, Deep structure of the Nagamachi-Rifu fault deduced from small aperture seismic array observations, Earth Planets 
Space, 54, 1033-1038, 2002.

Kulesh, M., M. S. Diallo, M. Holschneider, K. Kurennaya, F. Krüger, M. Ohrnberger, and F. Scherbaum, Polarization analysis in the wavelet domain based on the adaptive covariance method, Geophys. J. Int., 170(2), 667-678, 2007.

Moriya, H., Precise arrival-time detection of polarized seismic waves using the spectral matrix, Geophys. Prospect., 56, 667-676, 2008.

Moriya, H. and H. Niitsuma, Precise detection of P-wave in low S/N signal by using time-frequency representations of a triaxial hodogram, Geophysics, 61, 1453-1466, 1996.

Moriya, H., K. Nagano, and H. Niitsuma, Precise source location of AE doublets by spectral matrix analysis of triaxial hodogram, Geophysics, 59, 36-45, 1994.

Nakajima, J., T. Matsuzawa, A. Hasegawa, and A. Zhao, Threedimensional structure of $\mathrm{Vp}$, Vs and $\mathrm{Vp} / \mathrm{Vs}$ beneath northeastern Japan: Implication for arc magmatism and fluids, J. Geophys. Res., 106, 21843-21857, 2001.

Samson, J. C., Description of the polarization states of the vector pro- cesses: application to ULF magnetic fields, Geophys. J. Int., 34, 403419, 1973.

Soma, N., H. Niitsuma, and R. Baria, Estimation of deeper structure at the Soultz Hot Dry Rock field by means of reflection method using 3C AE as wave source, Pure Appl. Geophys., 150, 661-676, 1997.

Soma, N., H. Niitsuma, and R. Baria, Reflection technique in timefrequency domain using multicomponent acoustic emission signals and application to geothermal reservoirs, Geophysics, 67(3), 928-938, 2002.

Soma, N., H. Niitsuma, and R. Baria, Reflection imaging of deep reservoir structure based on three-dimensional hodogram analysis of multicomponent microseismic waveforms, J. Geophys. Res., 112, B11303, doi:10.1029/2005JB004216, 2007.

Umino, N., H. Ujikawa, S. Hori, and A. Hasegawa, Distinct S-wave reflectors (bright spots) detected beneath the Nagamachi-Rifu fault, NE Japan, Earth Planets Space, 54, 1021-1026, 2002.

H. Moriya (e-mail: hirokazu@ni2.kankyo.tohoku.ac.jp) 